\title{
HISTOPATHOLOGICAL IMAGE ANALYSIS FOR ORAL CANCER CLASSIFICATION BY SUPPORT VECTOR MACHINE
}

\author{
Yohannes Bekuma Bakare \\ Department of Electrical and Computer Engineering, \\ Wollega University, Ethiopia, Africa \\ yohbek@gmail.com \\ Kumarasamy $\mathrm{M}$ \\ Department of Computer Science, \\ Wollega University, Ethiopia, Africa \\ drmksamy115@gmail.com
}

Submitted: May, 10, 2021 Revised: July, 25, 2021 Accepted: Aug, 07, 2021

\begin{abstract}
Oral cancer is caused by the mutation of the cells in the lips or in the mouth. The incidence rate and prevalence rate of oral cancer are increasing worldwide. Recently, the Machine Learning (ML) approaches play a vital role in medical image diagnosis. They provide accurate and rapid evaluation of the analysis of histopathological images using supervised learning. In this study, three different modules are developed namely preprocessing, feature extraction and classification module. Initially, the raw histopathological image is given to the median filter for the removal of background noise in the preprocessing module. In the next module, the temporal features such as energy, entropy etc., are extracted from the color components of the filtered images. Finally, the classification is done by employing the Support Vector Machine (SVM) and K-Nearest Neighbour (KNN) to classify histopathological images as normal or abnormal. Results show that the SVM classifier is better than KNN for the classification of oral cancer. The classification accuracy on 1224 histopathological images has been improved to $98 \%$ by using SVM classifier as compared with the KNN results of $83 \%$.
\end{abstract}

Keywords: Oral cancer, histopathological images, medical image analysis, support vector machine, nearest neighbour classifier, supervised classification.

\section{INTRODUCTION}

The incidence rate and prevalence rate of oral cancer are increasing worldwide and many artificial intelligence algorithms have been developed to analyze medical images as well as other fields of technology. A dynamic Bayesian network is utilized for oral cancer in [1] using genomic data. The significant network nodes are identified by applying functional and topological analysis on genomic data. A softmax discriminant classifier is designed in [2] for classifying oral cancer using pathological reports, radiological studies and radiation reports.

A large scale image retrieval system is implemented in [3] for histopathological image analysis. A supervised kernel hashing is applied to compress the high dimensional image features into binary codes. Then, an efficient hash table is generated using these codes to retrieve the images. A dictionary learning method is described in [4] for histopathological image analysis. It is a low-complexity method which grades the diseases using the learned dictionaries under a sparsely constraint. 
A structural approach for histopathological images analysis is discussed in [5]. Instead of using a single graph, it uses a set of sub-graphs to represent the images through graph walking. Then the distribution of graph edges is used for the analysis. Two optimization techniques such as particle swarm optimization and bee colony approaches are hybridized in [6] for oral cancer classification. To detect the risk level of oral cancer, linear discriminant analysis is employed.

A neuro fuzzy inference system is designed in [7] for classifying oral cancer. It uses the inputs such as lesion size and number of nodes from the pathology reports to determine the stages at the earliest. Optical coherence tomography based oral cancer detection is discussed in [8]. It studies the feasibility of classification of oral cancer using optical coherence tomography. Color oral images are utilized in [9] for lesion detection. Texture and fractal features are extracted and Neural Network (NN) classifier is used for the classification.

An efficient neighbourhood component analysis based approach for classifying oral cancer is described in [10]. The features weights are estimated by a gradient descent estimator which is stochastic in nature. KNN, Random Forest $(\mathrm{RF})$ and SVM are employed and their performances are compared. Oral computed tomography images are used in [11] for classifying oral cancer. It uses fuzzy cmeans segmentation after preprocessing the images by anisotropic diffusion. After segmentation, first order features along with run length matrix features are extracted and SVM is employed for the classification.

Wavelet based approach is described in [12] for oral cavity cancer classification. The noises are removed by the wavelet approach and then Choas network outputs are utilized for the classification. A multi-parametric system for oral cancer classification is discussed in [13]. Features are extracted from clinical, imaging modality and genomic. All features are preprocessed and then correlation based selection is applied independently for feature selection. Then they are classified using SVM, NN, decision tree and RF.

A capsule network model is developed in [14] for classifying oral cancer. It is more robust to affine transformation and rotation. Gaussian blurring is employed as a preprocessing technique before segmentation by Otsu thresholding. Pretrained AlexNet, GoogleNet and ResNet50 are utilized in [15] for classifying oral cell carcinoma. It uses manually extracted region of interest region for the classification.

The recent development of deep learning for image classification is also used for oral cancer classification in [16]. It uses three pretrained architectures such as ResNet50, Inceptionv3 and VGG16 for the classification of histopathological images by the use of transfer learning through concatenation. An enhanced histopathological analysis is discussed in [17] for grading oral cell carcinoma. It is a two stage system and uses deep neural networks and preprocessing technique by wavelets. Though many advanced techniques available recently, in this paper, an efficient histopathological image analysis for oral cancer classification by SVM is presented.

\section{METHODS AND MATERIALS}

The proposed system consists of three primary stages; preprocessing, feature extraction and classification. Figure 1 depicts the complete flow of the oral cancer classification system. 
Int. J.Adv.Sig.Img.Sci, Vol.7, No.2, 2021

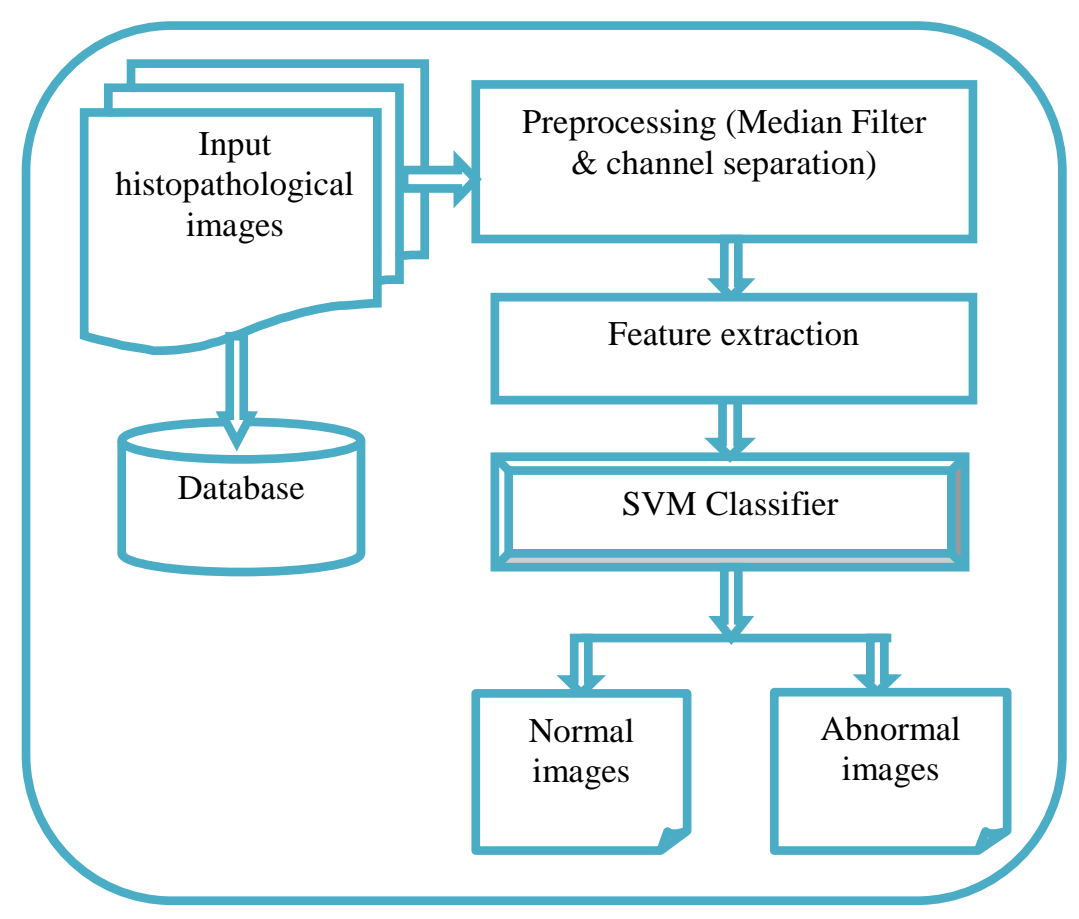

Fig. 1 Oral cancer classification system

The histopathological image is first preprocessed using median filter to eliminate the background noises followed by the separation of colour channels in the RGB dermoscopic images. Then, the pre-processed images are fed to the feature extraction phase, in which the unique temporal features are extracted from the skin images. Once the temporal features are extracted, SVM and KNN classifiers are used to classify the input into normal or abnormal.

\section{A. Pre-processing using Median Filter}

Initially the input dermoscopic images are preprocessed to remove unwanted hairs, background noise, and other noises. For this purpose, an efficient median filter algorithm with window filtering method is utilized. The median filter is a non-linear digital filtering method of reducing noise from an image or signal. Such noise reduction is a typical pre-processing technique to enhance edge detection on an image. Median filtering is extensively employed in digital image processing because it retains edges while eliminating noise. This filter method processes one color channel only. So the colour channels are separated at first.

Median filtering is a smoothing method that is good in removing noise from smooth patches or smooth regions of a picture, but it has a negative impact on edges. When reducing the noise in a signal, it's vital to keep the edges intact. The edge of a picture is one of the most important physical features of it. For small to moderate quantities of Gaussian noise, the median filter is superior to Gaussian blur in eliminating noise while retaining edges for a given window size. As a result, median filtering is frequently employed in digital image processing today. The pattern of neighbors is called the "window" and it glides, entry by entry, over the whole signal. Figure 2 shows the median filtered image and RGB colour components. 

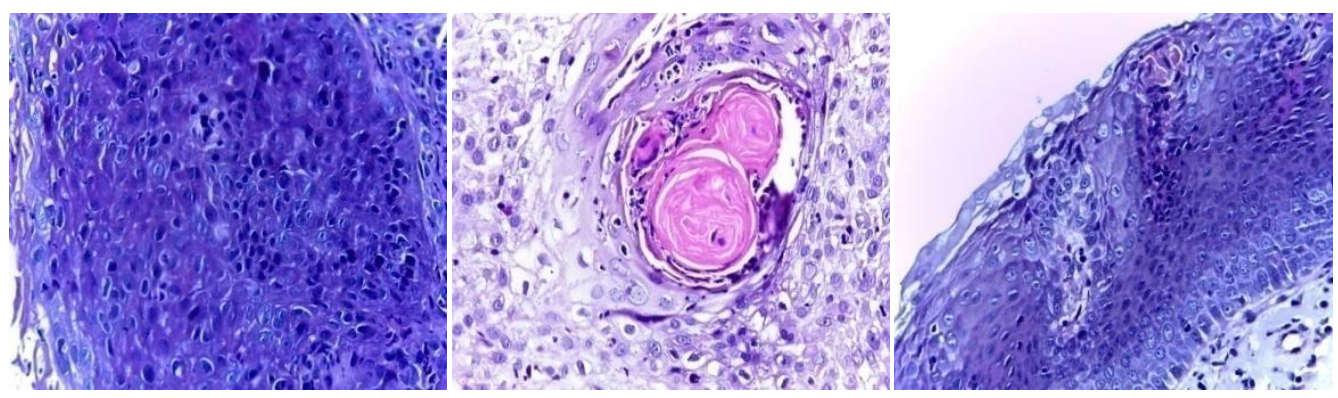

(a)
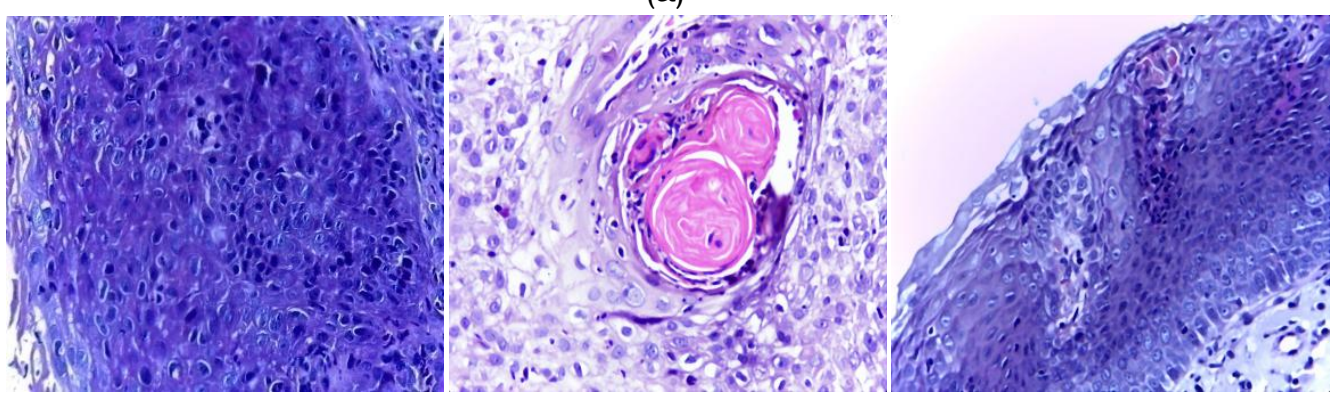

(b)

Fig. 2 Pattern recognition system for glaucoma diagnosis

\section{B. Feature Extraction}

The approach of extracting the features is an important phase in image processing. With huge data collection and in order to minimize the amount of resources without losing any significant or relevant information and also to decrease the quantity of duplicate information in a dataset, the feature extraction is employed. Reduced data helps to construct the model faster and with less effort on the part of the machine learning process. In the machine learning process, it is highly necessary to reduce the amount of datasets to develop the model faster and with less effort. Feature extraction of the temporal features such as entropy, energy, etc. Images were converted from RGB to gray scale images.

The term time domain refers to the study of signals in terms of time. The term "temporal features" can apply to any feature that is connected with or varies through time. When you have a sequence of photos taken at different times, may undergo temporal classification e.g. pixel in the case of imaging processing. Filtering is the most frequent time- or space-domain processing technique. Digital filtering usually involves a linear change of a number of surrounding samples that may be identified in terms of time or space. The output of a linear digital filter on any given input may be computed by convolving the input signal with an impulse response. But in the context of signal processing, a temporal feature might be any aspect of the data before it is converted into the Fourier, frequency or spectral domain, using the Fourier transform. In this system, the temporal features such as energy, entropy of individual colour components of red, green and blue components are extracted. Table 1 shows the temporal features formulae for feature extraction. 
TABLE 1 Temporal features Formulae for feature extraction

\begin{tabular}{|c|c|c|}
\hline Features & Energy & Entropy \\
\hline Formulae & $\frac{1}{R C} \sum_{i=1}^{R} \sum_{j=1}^{C}|\operatorname{skin}(i, j)|$ & $-\sum_{i} p_{i}{ }^{*} \log \left(p_{i}\right)$ \\
\hline \multicolumn{2}{|c|}{$\begin{array}{c}\text { Where, } R \text { is the width and } C \text { is the height of the input skin image and } \\
p_{i} \text { is the non-zero histogram value. }\end{array}$} \\
\hline
\end{tabular}

\section{SVM Classifier for Classification Module}

In this approach, the training dataset is used to train SVM about the classes, which is a typical supervised machine learning method for classification. A hyperplane in an $\mathrm{N}$-dimensional space $(\mathrm{N}=$ number of features) that clearly differentiates diverse input classes is the goal of the SVM method. When it comes to regression and classification, SVM is the go-to tool. If the data is not linearly separable, the kernels in nonlinear SVM convert it into high-dimensional space. SVM hyper parameter and model parameters must be optimized to get higher classification accuracy with less processing power. Figure 3 illustrates the SVM classifier with many hyperplanes and a maximum margin hyperplane.

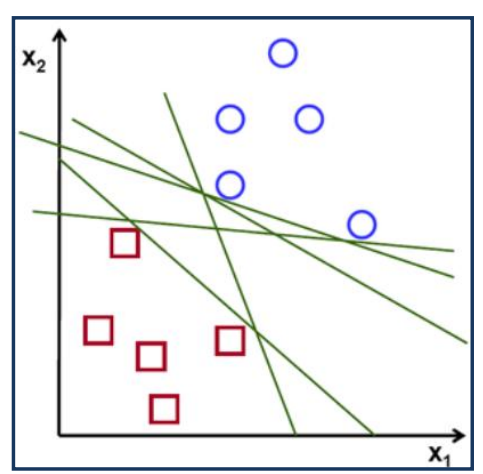

(a)

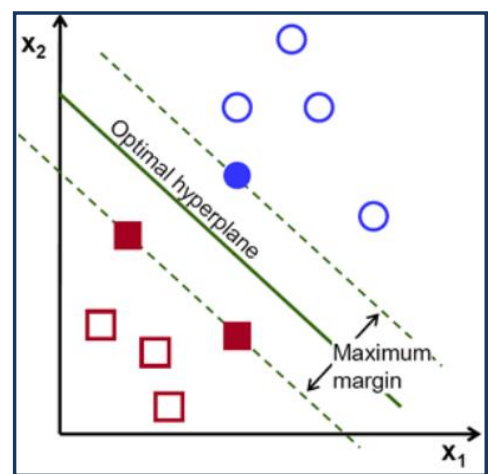

(b)

Fig. 3 SVM classifier (a) Many hyperplanes (b) Hyperplane with maximum margin

Figure 3(a) displays a number of hyperplanes that can be used to distinguish between the two classes of data. Margin is measured as the distance between the nearest dot positions in each class to the hyperplane. In this approach, a linear classifier that optimizes the distance between the hyperplane and the closest data point of each class is produced. The maximum margin classifier as shown in Figure 3(b) is a hyper-plane that is optimum for separating. It is possible to classify data points more accurately by increasing the margins. Therefore, the maximum margin classifier that optimally separates the two classes is one that greatly saves processing time. With the extracted temporal features and their classes, a trained SVM model must predict whether the given image is a normal image or melanoma skin cancer. A hyperplane can be found by separating various classes, weight vector ' $w$ ' and a bias ' $b$ ' are used. Hyperplane function can be represented as: 


$$
w^{T} x+b=0
$$

Depending on the value of the hyperplane function, the data points are classified as either normal or malignant and are expressed as:

$$
\begin{gathered}
\text { Normal if } \mathrm{w}^{\mathrm{T}} \mathrm{x}+\mathrm{b}<0 \\
\text { Cancerous if } \mathrm{w}^{\mathrm{T}} \mathrm{x}+\mathrm{b}>0
\end{gathered}
$$

When selecting the hyperplane, the maximum margin classification criterion is followed. The maximum margin classifier will always be parallel and equidistant, and the points on these two hyper-planes are referred to as support vectors. Data in high-dimensional space can be processed more efficiently using SVM since there is no over-fitting. Hence in the proposed system, the two stage SVM classification is done. In the SVM classifier, the sample images are identified as normal or abnormal images.

\section{RESULTS AND DISCUSSIONS}

In this system for the classification of the histopathological images, ML approach is utilized. Employing more than 1000 histopathological images (290 normal and 934 oral cancer images) from the database [18], the performance of the system is discussed in detail. In order to remove background noises and hairs, the image is first preprocessed using the intensity of colour channel separation technique. The images in the database are divided into two groups. Figure 4 shows the normal and oral cancer histopathological images.
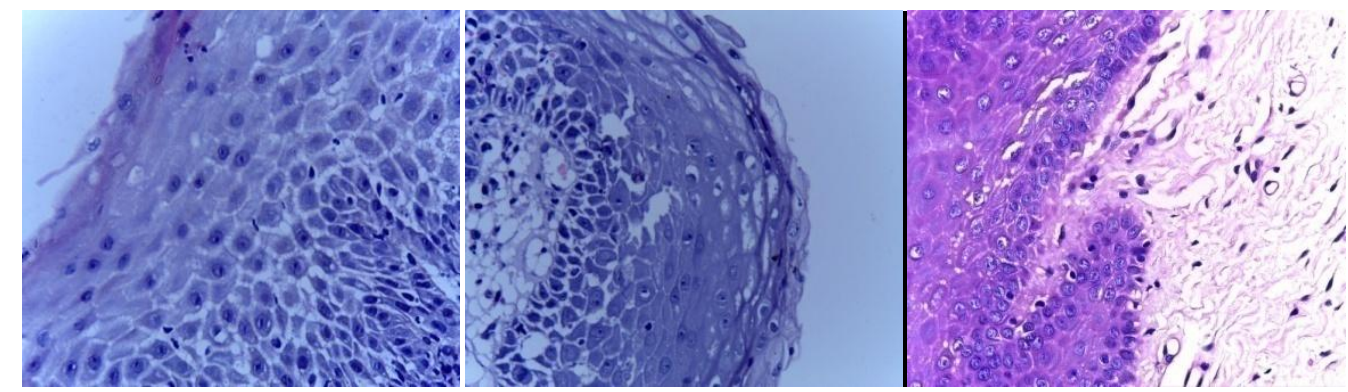

(a)
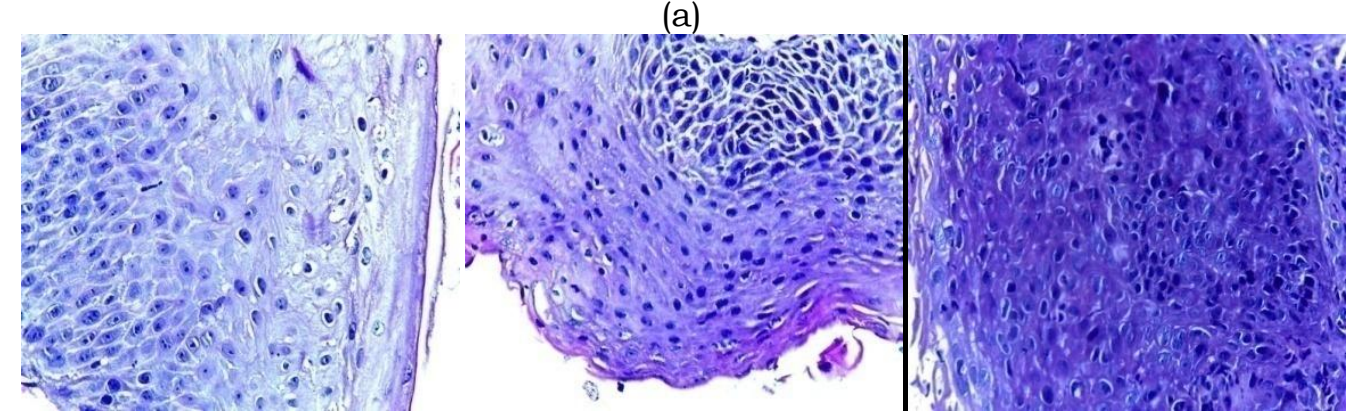

(b)

Fig. 4 Histopathological images (a) Normal (b) Oral cancer

After noise removal by median filter, the RGB colour channels of images are separated. Then the features are extracted from the skin lesion region using 
the images that have been processed. As soon as the temporal features have been extracted, The SVM classifier classifies the sample images as normal or abnormal. Further image analysis with melanoma characteristics is then conducted on the abnormal images, followed by the categorization of both malignant melanoma and non-malignant melanomas. As a result, in the proposed system, SVM classifier efficiency is evaluated and also compared with the KNN classifier performance. The confusion matrix derived from the 10 -fold cross-validation is used to evaluate the categorization system. The eqns. (3) to (5) gives the measures for accuracy, sensitivity, and specificity.

$$
\begin{aligned}
& \text { Accuracy }(\mathrm{ACC})=\frac{T P+T N}{T P+F N+T N+F P} \\
& \text { Specificity (SPE) }=\frac{T N}{T N+F P} \\
& \text { Sensitivity (SEN) }=\frac{T P}{T P+F N}
\end{aligned}
$$

where TP stands for True Positive; TN is for True Negative, whereas FP and FN stand for False Positive and False Negative. The Receiver Operating Characteristics (ROC) is also drawn for SVM and KNN classifier using the obtained $S P E$ and SEN. Figure 5 and Figure 6 show the performance of oral cancer classification system by KNN and SVM classifiers respectively.

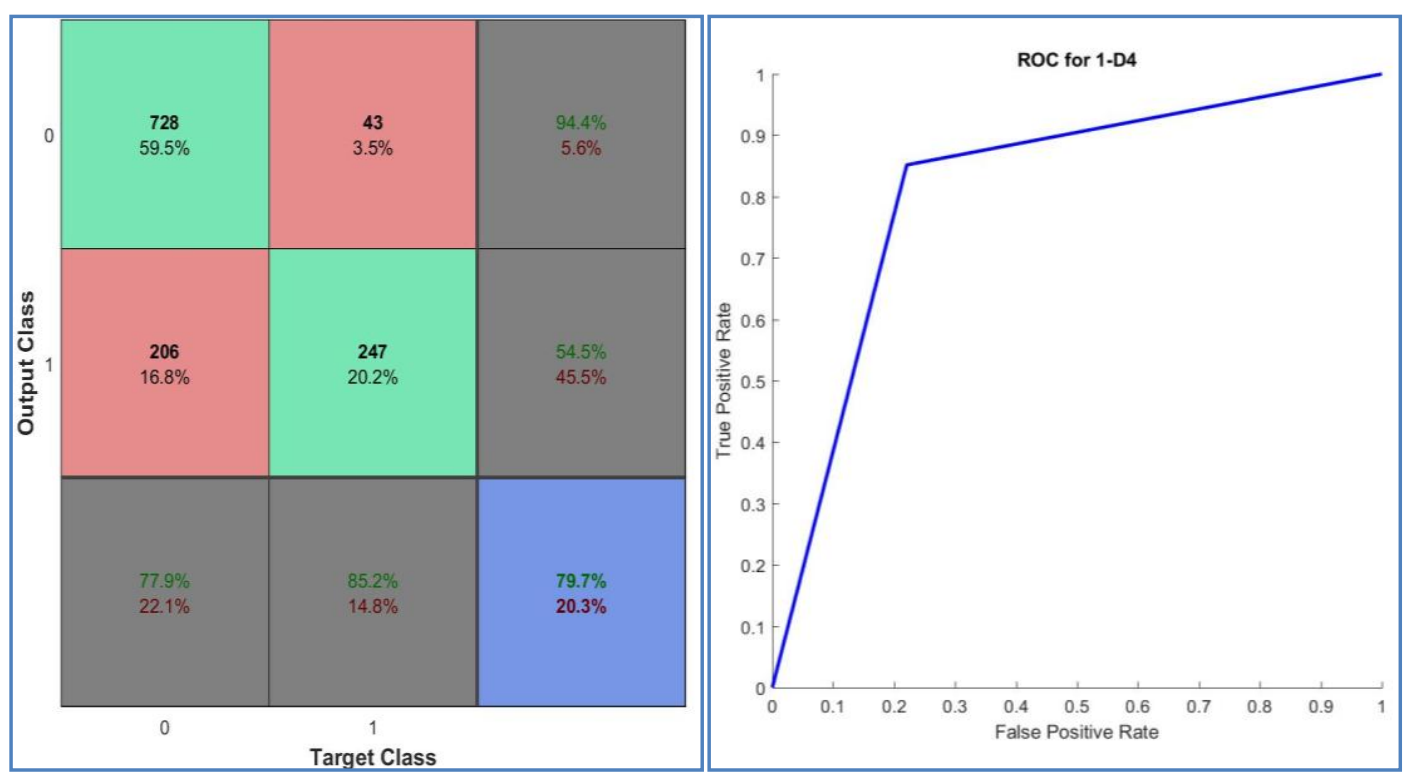

Fig. 5 Performance of KNN classifier; Confusion matrices (Left) and ROC (Right) 

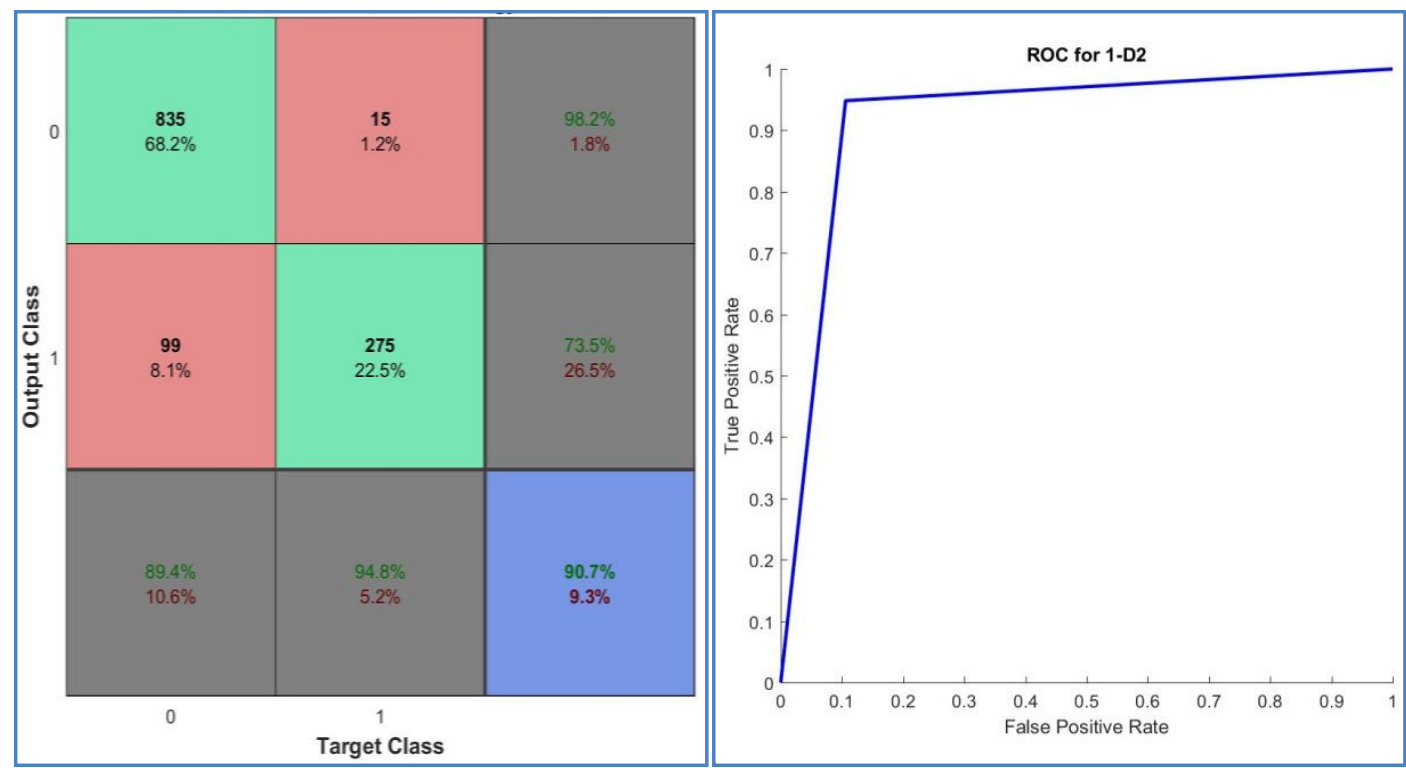

Fig. 6 Performance of SVM classifier; Confusion matrices (Left) and ROC (Right)

It can be seen from Figures 5 and 6 that the $S E N$ of the system has been improved by $12 \%$ by SVM classifier i.e., from $77.9 \%$ (KNN classifier) to $89.4 \%$. Also, the SPE of the system is $94.8 \%$ by SVM, whereas it is only 85.2 by KNN classifier. Also, it is noted from the ROCs of KNN and SVM is that the area under the curve by SVM (0.907) is more than KNN (0.797).

\section{CONCLUSION}

In this paper, an automated system with three phase module is presented for oral cancer classification. This system extracts the temporal features such as entropy, energy, and intensity values. These features are extracted from the individual Red, Blue and Green channel images that are obtained after filtering the dermoscopic images by the median filter in the preprocessing stage. Finally, the dermoscopic image classification is performed using SVM and KNN classifiers, the supervised ML model for diagnosing the oral cancer from histopathological images. The proposed system is evaluated on 1224 histopathological images and their performances are validated using the ground truth data. The confusion matrix result infers that SVM classifier provides more accuracy of $91 \%$ accuracy. KNN classifier provides only $80 \%$ of accuracy for oral cancer classification.

Funding Statement: The authors received no specific funding for this study.

Conflicts of Interest: The authors declare that they have no conflicts of interest to report regarding the present study. 


\section{REFERENCES}

[1]. K. Kourou, K. P. Exarchos, C. Papaloukas and D. I. Fotiadis, "A Bayesian Network-based approach for discovering oral cancer candidate biomarkers," International Conference of the IEEE Engineering in Medicine and Biology Society, 2015, pp. 7663-7666.

[2]. H. Rajaguru and S. Kumar Prabhakar, "An approach to classification of oral cancer using Softmax Discriminant Classifier," $2^{\text {nd }}$ International Conference on Communication and Electronics Systems, 2017, pp. 420423.

[3]. X. Zhang, W. Liu, M. Dundar, S. Badve and S. Zhang, "Towards LargeScale Histopathological Image Analysis: Hashing-Based Image Retrieval," IEEE Transactions on Medical Imaging, vol. 34, no. 2, 2015, pp. 496-506.

[4]. T. H. Vu, H. S. Mousavi, V. Monga, G. Rao and U. K. A. Rao, "Histopathological Image Classification Using Discriminative FeatureOriented Dictionary Learning," IEEE Transactions on Medical Imaging, vol. 35, no. 3, 2016, pp. 738-751.

[5]. G. Olgun, C. Sokmensuer and C. Gunduz-Demir, "Graph walks for classification of histopathological images," $10^{\text {th }}$ International Symposium on Biomedical Imaging, 2013, pp. 1126-1129.

[6]. H. Rajaguru and S. Kumar Prabhakar, "Oral cancer classification from hybrid ABC-PSO and Bayesian LDA," $2^{\text {nd }}$ International Conference on Communication and Electronics Systems, 2017, pp. 230-233.

[7]. A. Pandey and N. K. Gupta, "Stage determination of oral cancer using neurofuzzy inference system," IEEE Students' Conference on Electrical, Electronics and Computer Science, 2014, pp. 1-5.

[8]. Woonggyu Jung et al., "Advances in oral cancer detection using optical coherence tomography," IEEE Journal of Selected Topics in Quantum Electronics, Vol. 11, No. 4, pp. 811-817, 2005.

[9]. N. B.R., G. Kiran A., D. M.S., M. S. and C. H.S., "Texture Analysis of Color Oral Images for Lesion Detection," International Conference on Computational Performance Evaluation, 2020, pp. 067-072.

[10]. A. Nawandhar, N. Kumar and L. Yamujala, "Performance Analysis of Neighborhood Component Feature Selection for Oral Histopathology Images," PhD Colloquium on Ethically Driven Innovation and Technology for Society, 2019, pp. 1-8.

[11]. Z. Hu, A. Alsadoon, P. Manoranjan, P. W. C. Prasad, S. Ali and A. Elchouemic, "Early stage oral cavity cancer detection: Anisotropic preprocessing and fuzzy C-means segmentation," $8^{\text {th }}$ Annual Computing and Communication Workshop and Conference, 2018, pp. 714-719.

[12]. Y. Hu, Z. Zhao and T. Jiang, "Classification of Squamous Cell Carcinoma of the Oral Cavity Using Wavelet Analysis and BP-Chaos Networks," First International Conference on Information Science and Engineering, 2009, pp. 3613-3616.

[13]. K. P. Exarchos, Y. Goletsis and D. I. Fotiadis, "Multiparametric Decision Support System for the Prediction of Oral Cancer Reoccurrence," IEEE Transactions on Information Technology in Biomedicine, Vol. 16, No. 6, pp. 1127-1134, 2012.

[14]. S. Panigrahi, J. Das, and T. Swarnkar, "Capsule network based analysis of histopathological images of oral squamous cell carcinoma," Journal of King Saud University-Computer and Information Sciences. 2020, pp.1-8.

[15]. D. Kadaru and B. Brahmeswara, "Automated classification of Oral Squamous cell carcinoma stages detection using Deep Learning 
Techniques," European Journal of Molecular \& Clinical Medicine, Vol. 7, No. 4, 2020, pp.1111-1119.

[16]. I. Amin, H. Zamir, and F.F. Khan, "Histopathological Image Analysis for Oral Squamous Cell Carcinoma classification using concatenated deep learning models," medRxiv, 2021, pp.1-10.

[17]. J. Musulin, D. Stifanic, A. Zulijani, T. Cabov, A. Dekanic, and Z. Car, "An enhanced histopathology analysis: An AI-based system for multiclass grading of oral squamous cell carcinoma and segmenting of epithelial and stromal tissue," Cancers, Vol. 13, No. 8, 2021, pp. 1784-2005.

[18]. T.Y Rahman, L.B. Mahanta, A.K. Das and J.D. Sarma, "Histopathological imaging database for oral cancer analysis,", Data in brief, Vol. 29, 2020. pp.1-18. 